\title{
Aspectos químicos e atividade antibacteriana de Piptadeniagonoacantha (FABACEAE)
}

\section{Chemical aspects and antibacterial activity of Piptadeniagonoacantha (Fabaceae)}

Camilo Amaro de Carvalho*1, Gabriela Silva Santana ${ }^{2}$, Marilene de Oliveira Fani Amaro ${ }^{1}$, Luciana Moreira Lima ${ }^{1}$, Fernanda Brum Pires ${ }^{3}$, Valéria Dal Prá ${ }^{3}$, Silvia A. Cardoso ${ }^{1}$, Marcelo Barcellos da Rosa ${ }^{3,4}$, Leandro Licursi de Oliveira ${ }^{5}$

${ }^{1}$ Departamento de Medicina e Enfermagem, Universidade Federal de Viçosa, Brasil.

${ }^{2}$ Departamento de Farmácia, União de Ensino Superior de Viçosa, Brasil.

3Programa de Pós Graduação em Ciências Farmacêuticas, Universidade Federal de Santa Maria, Brasil. ${ }^{4}$ Departamento de Química, Universidade Federal de Santa Maria, Universidade Federal de Santa Maria, Brasil.

${ }^{5}$ Departamento de Biologia Geral, Universidade Federal de Viçosa, Brasil.

\begin{abstract}
Resumo
Este trabalho teve o objetivo de avaliar os aspectos fitoquímicos e antibacteriano de Piptadeniagonoacantha. Inicialmente, realizou-se uma padronização dos extratos das folhas de P. gonoacantha e sua caracterização fotoquímica foi analisada, onde as principaisvariáveis avaliadas foram: método de extração (maceração e banho de ultrassom), solvente (água, álcool e misturas hidroalcoólicas), temperatura de extração $\left(30,40\right.$ e $50^{\circ} \mathrm{C}$ ) e influência do $\mathrm{pH}(2$ a 12) sobre o coeficiente de extinção (absorbância) das mesmas. Além disto, foi realizada uma prospecção fitoquímica e a quantificação de polifenóis totais na amostra. Umaavaliação da atividade antibacteriana in vitro foi realizada através da adaptação do método de difusão em meio sólido com perfuração do ágar frente aStaphylococcus aureus e Escherichia coli. Por meio da avaliação química identificou-se a maneira mais eficiente de promover a extração dos seus constituintes, devendo esta ser realizada por ultrassom, a $40^{\circ} \mathrm{C}$, utilizando etanol $80 \%$ (v/v). O PHpromoveu modificaçõesestruturais (desprotonação/protonação) dos constituintes químicos em extratos com valores de $p H$ acima de 8. Além disso, a quantificação de fenóis totais indicou que grande proporção dos extratos é constituída por compostos incluídos nesta classe, sendo confirmada pela prospecção fitoquímica onde pôde ser evidenciada a presença de flavonoides, taninos, cumarinas e antraquinonas. O extrato desta espécie apresentou atividade antibacteriana, especialmente com etanol $80 \%(v / v)$, tendo eficiente capacidade de inibir o crescimento da bactéria S. aureus. Os dados obtidos sugerem que a espécie P. gonoacantha possui compostos promissores como fonte para obtenção de novos medicamentos no combate a microrganismos resistentes. Palavras-chave: Piptadeniagonoacantha, plantas medicinais, antibacteriano, fitoquímica.
\end{abstract}

\begin{abstract}
This study aimed to evaluate the antibacterial and phytochemical aspects of Piptadeniagonoacantha . First, there was a standardization of extracts from the leaves of $P$. gonoacantha and its phytochemical characterization were analyzed, where the main variables were: extraction method (maceration and ultrasound bath), solvent (water, alcohol and water-alcohol mixtures), extraction temperature (30, 40 and $\left.50^{\circ} \mathrm{C}\right)$ and influence $\mathrm{pH}$ (2 to 12) on the extinction coefficient (absorbance) of the same. Moreover, a phytochemical screening and quantification of total polyphenols in the sample was performed. An evaluation of the antibacterial activity in vitro was performed by adapting the method of diffusion in solid media with perforation of agar against Staphylococcus aureus and Escherichia coli. Through chemical evaluation identified the most effective way to promote the extraction of constituents should be performed with ultrasound at $40^{\circ} \mathrm{C}$ using $80 \%$ ethanol (v/v). The pH promoted structural modification (Deprotonation/protonation) of chemical constituents in extracts with $p H$ values above 8 . Furthermore, quantification of total phenols indicated that a significant proportion of the extracts is constituted by compounds within this class is confirmed by phytochemical which could be evidenced the presence of flavonoids, tannins, coumarinsandanthraquinones. The extract of this species showed antibacterial activity, especially with $80 \%$ ethanol (v/v), and efficient ability to inhibit the growth of bacteria S. aureus . The data suggest that the species P. gonoacantha has promising compounds as their source of new drugs to combat resistant microorganisms. Keywords: Piptadeniagonoacantha, medicinal plants, antibacterial, phytochemical.
\end{abstract}

"camilo.carvalho@ufv.br

Recebido: 08/04/2014 Aceito: 08/04/2014 


\section{Introdução}

$\mathrm{O}$ uso de plantas como medicamento não é um fato recente, surgiu na era primitiva, onde o ser humano, por experimentação e observação, descobria as propriedades das plantas. Porém, somente na primeira década do século $X X$ a quimioterapia surgiu como ciência. No ano de 1920, Alexander Fleming descobriu o primeiro antibiótico, a penicilina, a partir do fungo Penicilliumnotatum. Essa descoberta permitiu o desenvolvimento de outros compostos antimicrobianos produzidos por organismos vivos (Peres et. al., 2009).

Os termos "antibiótico" e "antimicrobiano" são considerados como sinonímia, indicando, assim, toda substância originária de seres vivos, microrganismos ou vegetais, como também aquelas sintetizadas em laboratório com a capacidade de apresentarem atividade letal ou inibitória contra espécies microbianas em pequenas concentrações (Amato Neto et. al., 1994; Ribeiro e Soares, 2008). Embora as indústrias químicas e farmacêuticas tenham produzido uma enorme variedade de diferentes antibióticos nos últimos tempos, cada vez mais se tem observado o aumento de microrganismos resistentes aos antimicrobianos disponíveis no mercado, o que incentiva a busca de novas fontes de substâncias com atividades antimicrobianas (Ribeiro e Soares 2008). Entretanto, o controle de qualidade de fitoterápicos é imprescindível no meio comercial, pois muitas espécies vegetais são vendidas sem qualquer garantia de qualidade, favorecendo desde a venda de espécies falsificadas até o armazenamento inadequado durante a sua comercialização (Carvalho et. al., 2008).

Diante desta realidade, torna-se fundamental a sistematização de técnicas para uma melhor comprovação científica da real utilização dos fitoterápicos e validação dos processos tecnológicos de cultivo e produção.

A escolha de um procedimento adequado, capaz de identificar estruturalmente as inúmeras moléculas que podem vir a constituir uma espécie vegetal, é um ponto fundamental no estudo de princípios ativos (Carvalho et. al., 2008).

Dentre os procedimentos e técnicas empregados para a validação e/ou elucidação de compostos, destaca-se a espectrometria molecular na região ultravioleta-visível (UV-VIS). Esta é uma técnica analítica que vem sendo há mais de 50 anos empregada para a identificação e determinação qualitativa e quantitativa de muitas espécies moleculares inorgânicas e orgânicas em diferentes tipos de materiais (Peres et. al., 2009).

Além da escolha adequada do procedimento e técnica eficaz no processo de avaliação de amostras, os solventes destacam-se como um fator fundamental, uma vez que estes irão influenciar na solubilidade das amostras e, assim, nos resultados das análises (Simões et.al., 2004).

Por influenciar diretamente nos resultados finais, a solubilidade tem extrema importância no método de extração, já que ela determina a quantidade máxima de soluto que pode ser solubilizada nas condições operacionais (Simões et. al., 2004). Assim, quanto maior for à solubilidade do soluto maior será a quantidade de produto extraído. Por ser uma propriedade termodinâmica, a solubilidade depende da pressão, temperatura, natureza e composição do solvente.

Os experimentos realizados neste trabalho tiveram como objetivo avaliar aspectos químicos da espécie $\mathrm{P}$. gonoacantha com relação às variações do perfil espectral dos extratos de suas folhas, decorrentes das diferentes condições a que foram submetidos durante o processo de extração. Avaliou-se também a influência do PH nestes espectros e determinou-se o teor de compostos fenólicos totais. Além da avaliação da atividade antibacteriana in vitro, frente à S. aureus e E. Coli.

\section{Materiais e métodos}

\subsection{Aspectos botânicos - material botânico}

Foram coletadas plantas da espécie Piptadeniagonoacantha, conhecida popularmente como Pau de Jacaré, no município de Viçosa, Minas Gerais - Brasil. O material coletado foi identificado e autenticado por comparação com espécies do Horto Botânico da Universidade Federal de Viçosa (UFV), onde a espécie testemunho foi depositada (exsicata $\mathrm{n}^{\circ} 35.530$ ).

\subsection{Aspectos químicos - preparo do extrato:}

Os extratos de Piptadeniagonoacantha foram preparados, separadamente, a partir da utilização de folhas na proporção de $20 \%$ (m/v - pulverizado da planta/solvente). Antes do preparo dos extratos as folhas foram secas em estufa de ar circulante $40 \pm 2^{\circ} \mathrm{C}$ e trituradas em moinho de facas Marconi ${ }^{\circledR}$ (Carvalho et al., 2011). Para as análises espectrofotométricas utilizaram-se os extratos diluídos na concentração de $1 \%(\mathrm{~m} / \mathrm{v}$ - extrato da planta/solvente). Os espectros foram medidos no espectrômetro FEMTO $800 \mathrm{XI}{ }^{\circledR}$, com cubeta de quartzo de $1,0 \mathrm{~cm}$ de caminho óptico, intervalos de $2 \mathrm{~nm}$ e varreduras de 200 a $700 \mathrm{~nm}$. Para o branco foi empregado o solvente

\section{Influência do PH:}

Para os estudos de protonação e desprotonação do extrato aquoso $(20 \% \mathrm{~m} / \mathrm{v})$ da espécie P. gonoacantha, os extratos foram preparados e diluídos a $1 \%(\mathrm{~m} / \mathrm{v}) \mathrm{em}$ volumes definidos de $\mathrm{NAOH} 0,01 \mathrm{M}$ ou $\mathrm{HCl} 0,01 \mathrm{M}$ para avaliar a influência do $\mathrm{pH}$ sobre os extratos, sendo este medido potenciometricamente (PHmetroQuimis ${ }^{\circledR}$ - Q 400A), com eletrodo de. Os espectros foram medidos no espectrômetro FEMTO ${ }^{\circledR}$ 800XI. Os extratos foram analisados na faixa de $\mathrm{PH}$ entre 2 e 12, considerando os dez pontos compreendidos nesta. 
Tabela 1. Variáveis no processo de preparo de extratos de P. gonoacantha.

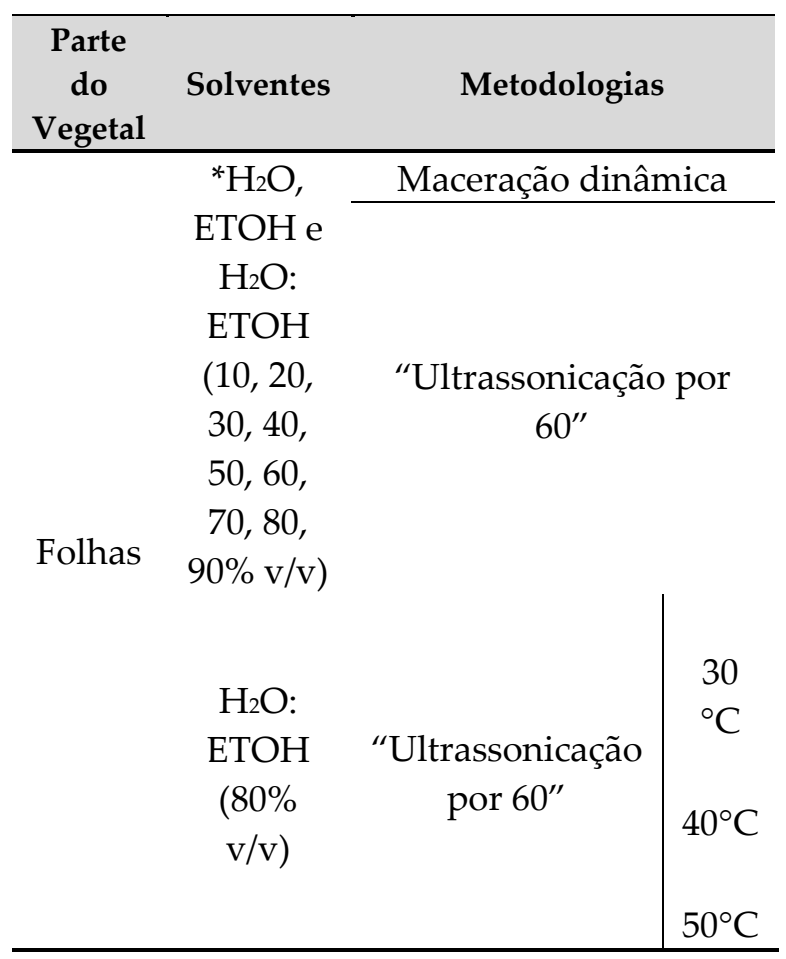

\section{Estudo da prospecção Fitoquímica}

Os extratos foram submetidos às analises fitoquímicos para a caracterização de: alcaloides, antraquinonas, taninos, flavonoides, cumarinas, heterosídeos cardiotônicos, saponinas, terpenos e compostos fenólicos. As análises foram realizadas por meio de reações cromáticas e de precipitação, segundo metodologia descrita em Simões et. al. (2004).

\section{Quantificação de Polifenóis Totais}

Polifenóis totais foram quantificados em todos os extratos, preparados nas mesmas condições. Para a avaliação do conteúdo de fenólicos totais solúveis, utilizou-se o método colorimétrico Folin-Ciocalteau. Foram mantidas as proporções estabelecidas segundo a RDC no313 (Brasil 2005).

O teor de compostos fenólicos foi determinado por espectrofotometria no visível, construindo-se uma curva analítica utilizando padrão de ácido tânico 32, 25,6, 19,2, $12,8,6,4$ e 3,2 $\mu \mathrm{g}$.mL-1. A equação obtida pela regressão linear da curva de calibração foi utilizada para expressão dos valores. A absorbância das amostras e amostra-padrão foram medidas em espectrofotômetro $(\lambda=750 \mathrm{~nm})$, exatamente 2 minutos após a adição do último reagente. Água destilada foi utilizada como branco. Os resultados foram expressos como mg de ácido gálico equivalentes (GAE) por grama de extrato (mg de GAE/g). Os experimentos foram realizados em triplicata. A Equação da curva de calibração do ácido gálico foi $\mathrm{y}=0,0062 \mathrm{x}+$ 0,2623 e o coeficiente de correlação $R 2=0,9899$. Todas as análises foram realizadas em triplicata.

\subsection{Aspectos biológicos - avaliação microbio- lógica preparo do meio de cultura e das bacté- rias:}

A análise da atividade antibacteriana da espécie P. gonoacantha foi realizada através da adaptação do método de difusão em meio sólido com perfuração em ágar. Os microrganismos utilizados nos testes foram obtidos no Laboratório de Imunobiologia Molecular e Glicobiologia da Universidade Federal de Viçosa - MG. Utilizou-se uma linhagem de Staphylococcus aureus (ATCC 29213) e Escherichia coli (ATCC 14948).

No ensaio foi empregado o meio de cultura Mueller -Hinton(Himedia®, Lote: 26206), preparado segundo as especificações do fabricante. As culturas de bactérias foram mantidas a $4^{\circ} \mathrm{C}$ em Mueller-Hinton. Antes dos testes, as linhagens foram repicadas para o meio citado e incubadas a $36 \pm 2{ }^{\circ} \mathrm{C}$ por 24 horas. A partir de culturas recentes, foram preparadas suspensões bacterianas em solução salina NACl 0,9\% com turvação equivalente a Escala de McFarland 0,5 (1,5 x 108 células/mL) (NCCLS 2003). Foi realizado controle da absorbância destas suspensões de microrganismos; sendo a leitura realizada em espectrofotômetro ajustado para o comprimento de onda de $600 \mathrm{~nm}$.

Posteriormente, $500 \mu \mathrm{L}$ da suspensão do microrganismo foram misturadas ao meio Mueller-Hinton estéril, em estado líquido $(12,5 \mathrm{~mL})$ a $37^{\circ} \mathrm{C}$, sendo em seguida vertido em placas de Petri estéreis (diâmetro $90 \mathrm{~mm}$ ). Os poços foram confeccionados utilizando-se uma bomba a vácuo acoplada a uma ponteira estéril previamente adaptada para tal fim. Em cada placa foram perfurados treze poços para aplicação das amostras.

Foram depositados $10 \mu \mathrm{L}$ dos extratos e dos controles nas cavidades correspondentes a cada um. Após a incubação por 24 horas, em estufa a $37^{\circ} \mathrm{C}$, o diâmetro do halo de inibição foi mensurado em $\mathrm{mm}$.

Posteriormente, $500 \mu \mathrm{L}$ do extrato $(20 \% \mathrm{~m} / \mathrm{v})$ foram concentrados em estufa a $40^{\circ} \mathrm{C}$; até volume final fixado em $200 \mu \mathrm{L}(50 \% \mathrm{~m} / \mathrm{v})$. Uma alíquota de $10 \mu \mathrm{L}$ de cada extrato foi inoculada em cada poço confeccionado nas placas contendo o meio Mueller-Hinton. Após a incubação por 24 horas, em estufa a $37^{\circ} \mathrm{C}$, o diâmetro do halo de inibição foi mensurado em $\mathrm{mm}$, sendo os resultados organizados e descritos pela estatística descritiva.

Atividade antibacteriana e determinação da concentração inibitória mínima

Todos os testes foram realizados em triplicata, sendo considerado como resultado final de cada extrato a média das três medidas e como susceptível halo igual ou acima de oito $\mathrm{mm}$ de diâmetro (Parekhet al., 2007; Santos et.al. 2007).

Para determinação da concentração inibitória mínima 
(CIM), a avaliação da atividade antimicrobiana foi realizada observando-se a formação de halos de inibição ao redor das cavidades padronizadas. Sendo considerada a CIM como a mais alta diluição de EPG que inibe o crescimento dos microrganismos (NCCLS 2012).

Foi verificada também a atividade antibacteriana do álcool utilizado para a preparação dos extratos, onde cada cavidade recebeu $10 \mu \mathrm{L}$ água e de álcool etílico PA (Lafan Química Fina ${ }^{\circledR}$ ) em todas as graduações alcoólicas, além do controle positivo Eritromicina $10 \mu \mathrm{g}$. $\mu \mathrm{L}-1$ (Lote: 080616451 - Pharmanostra ${ }^{\circledR}$ ) para S. aureus

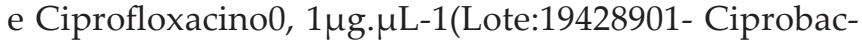
ter®) para E. Coli.

\section{Resultados e discussão}

A técnica de extração por ultrassom apresentou maiores valores de coeficiente de extinção (absorbância) ao longo dos comprimentos de onda analisados, indicando ser o método de maior eficiência na extração das folhas da espécie Piptadeniagonoacantha.

Tomando-se, como exemplo, seis comprimentos de onda, 250, 270, 291, 311, 331, $351 \mathrm{~nm}$ (Tabela 3), escolhidos por serem os comprimentos de onda onde são analisadas as maiores absorbâncias ao longo dos espectros, podese comparar a proporção dos compostos extraídos por cada metodologia.

Pelo seu caráter, a técnica de maceração não conduz ao esgotamento da matéria-prima vegetal, seja devido à saturação do líquido extrator ou ao estabelecimento de um equilíbrio difusional entre o meio extrator e o interior da célula vegetal, neste caso (Vinatoruet al., 1997). Entretanto, o banho de ultrassom pode facilitar a dilatação e hidratação do material da planta e

Tabela 3. Influência do método de extração sobre coeficiente de extinção (absorbância) dos extratos de Piptadeniagonoacantha.

\begin{tabular}{ccc}
\hline \multirow{2}{*}{$\lambda / \mathbf{n m}$} & \multicolumn{2}{c}{ Absorbância (nm) } \\
\cline { 2 - 3 } & Ultrassom & Maceração \\
\hline $\mathbf{2 5 0}$ & $2,990 \pm 0,17$ & $1,871 \pm 0,11$ \\
$\mathbf{2 7 0}$ & $2,990 \pm 0,28$ & $2,380 \pm 0,11$ \\
$\mathbf{2 9 1}$ & $2,990 \pm 0,15$ & $1,720 \pm 0,08$ \\
$\mathbf{3 1 1}$ & $2,990 \pm 0,12$ & $0,957 \pm 0,10$ \\
$\mathbf{3 3 1}$ & $1,922 \pm 0,11$ & $0,704 \pm 0,08$ \\
$\mathbf{3 5 1}$ & $1,573 \pm 0,10$ & $0,614 \pm 0,12$ \\
\hline
\end{tabular}

Segundo Franconyet al. (1996), a extração por ultra-sonificação apresenta-se como técnica bastante robusta. Sendo que os efeitos físicos provocados pelos ultrassons ocorrem devido ao fenômeno da cavitação acústica, que é o processo de nucleação, crescimento e colapso de bolhas transientes em líquidos expostos a ondas ultrassônicas de baixa frequência(< $1 \mathrm{MHz})$. A energia liberada durante a cavitação acústica fornece excelentes perspectivas para o preparo e/ou tratamento de amostras (Kornet al., 2005; Suslick 1990). No preparo de amostras, o colapso das microbolhas favorece a extração de espécies químicas a partir de materiais sólidos, bem como a dissolução destes (Ruiz-Jiménez et. al., 2003; Nascentes et. al., 2001).

Com relação aos diferentes gradientes hidroalcoólicos utilizados, pode-se observar que os extratos apresentaram perfis espectrais semelhantes, porém com extração de moléculas em quantidades diferentes (Figura 1A). Cada extrato apresentou uma faixa de comprimento de onda específico com absorbâncias em níveis equivalentes à concentração dos metabólitos extraídos nos respectivos comprimentos de ondas correspondentes ao coeficiente de partição de cada solvente extrator.

As diferenças espectrais analisadas estão intimamente relacionadas com a diferença no gradiente hidroalcóolico utilizado no processo de extração. Onde, ao utilizar o etanol a 80, 70, 60, 50 e 40\% (v/v) como solvente, houve uma maior extração dos constituintes globais de folhas da espécie P. gonoacantha, seguidos respectivamente pelo etanol 30, 90, 20, 100, 10 e 0\% (v/v). Cabe ressaltar que estes valores são mantidos ao longo de todo o perfil espectral, exceto para os constituintes que absorvem nos comprimentos de onda acima de $370 \mathrm{~nm}$, extraídos com o solvente hidroalcoólico a $100 \%$ (v/v).

Os maiores valores de absorvências observadas na extração utilizando o etanol a $80 \%(\mathrm{v} / \mathrm{v})$ podem estar relacionados à maior concentração de metabólitos com polaridade semelhante à deste solvente. De acordo com Falkenberget al. (2003) a grande maioria dos constituintes de interesse para a análise fitoquímica apresenta alguma solubilidade em misturas etanoicas ou metanólicas a $80 \%$, de tal modo que estas costumam ser empregadas com frequência. Essa observação é compartilhada por Ferri (1996). Este afirma que, a partir de extratos etanólicos, podem ser realizadas várias extrações, caso se deseje obter frações específicas como alcalóides e flavonóides, por exemplo. Além disso, segundo o mesmo autor, a utilização de etanol nessa concentração é mais barata, rápida e atóxica para pesquisas dessa natureza.

O extrato das folhas, utilizando etanol $80 \%$ (v/v) como solvente, foi selecionado para dar continuidade na análise da influência da temperatura em relação ao processo de extração e atividades biológicas, por este extrato apresentar valores elevados de absorbância dos constituintes. Este extrato, quando submetido às diferentes temperaturas de extração, apresentou 
a $40^{\circ} \mathrm{C}$ o maior coeficiente de extinção em todos os comprimentos de onda analisados, demonstrando ser a melhor temperatura para a extração dos constituintes globais desta espécie (Figura 1B e C).

De maneira geral, a temperatura de extração é um parâmetro a ser aperfeiçoado, a fim de diminuir custo do processo de extração. Alguns autores, embora concordem que a temperatura poderá favorecer a extração de alguns compostos, dentre eles os fenólicos, ressaltam que também poderá desencadear a sua degradação com possível prejuízo da ação antioxidante (Spignoet al., 2007; Yilmaz et. al., 2006; Pinelo et. al., 2005).

Medidas de deslocamento batocrômico espectral em função da deprotonação forçada (adição de concentrações e/ou gotas conhecidos de $\mathrm{NaOH}$ ) e formação de pontos isosbésticos (Skoog et al., 2002) em função do pH foram obtidos para todos os extratos aquosos das folhas de P. gonoacantha. Por meio dos resultados é possível verificar uma mudança significativa no perfil espectral do extrato aquoso das folhas de P. gonoacantha, para valores de $\mathrm{pH}>8,0$ (Figura 1D). O que indica uma plausível deprotonação de alguns grupamentos cromóforos da espécie P. gonoacantha. Espectros que foram medidos para valores de $\mathrm{pH}$ entre 2,0 e 7,0, não apresentaram modificações em suas estruturas, indicando uma protonação molecular para esta faixa de $\mathrm{pH}$, bem como espectros medidos para valores de $\mathrm{pH}$ $>$ 9. Estes mantiveram o mesmo perfil espectral sendo, portanto, evidenciado apenas o último valor de $\mathrm{pH}$ analizado ( $\mathrm{pH} 12)$.
A

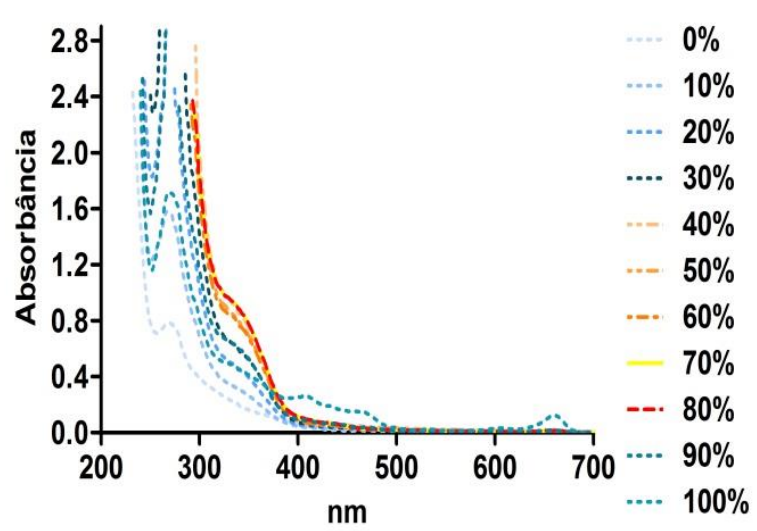

D

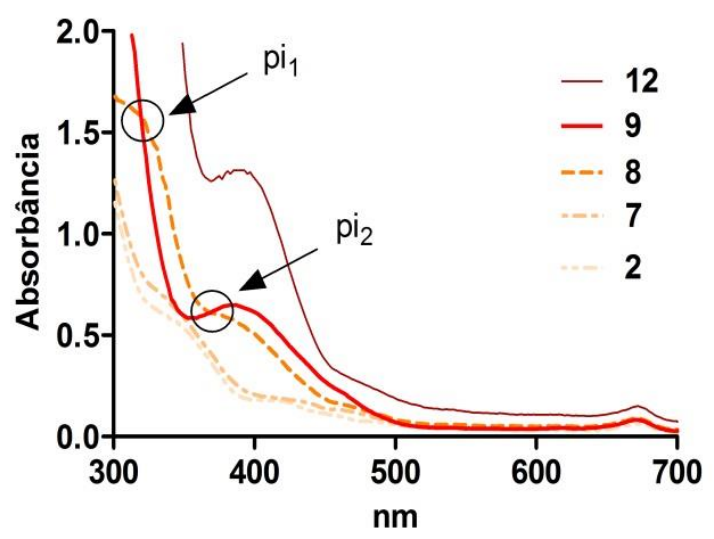

B

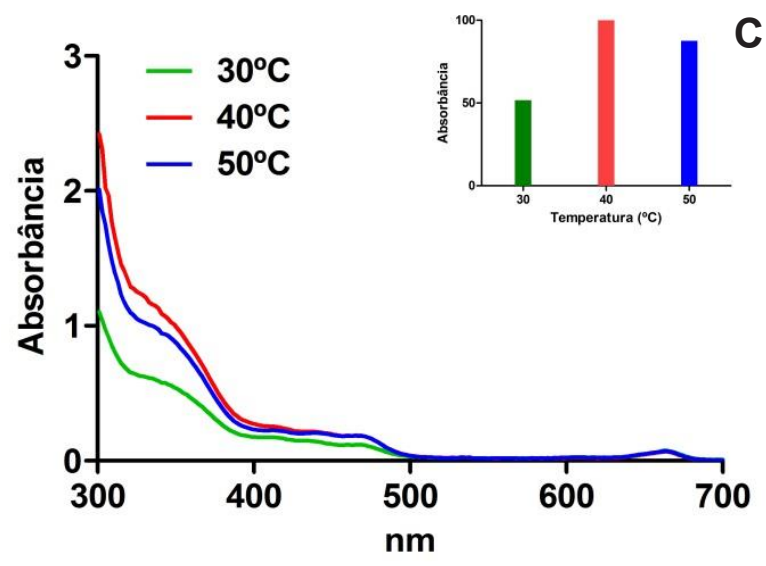

E

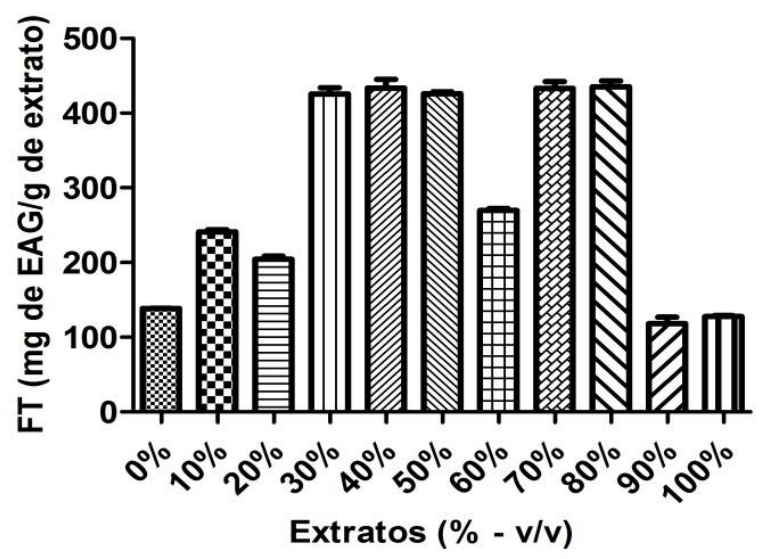

Figura 1.Análises espectrofotométricas dos extratos de folhas de Piptadeniagonoacantha(EPG).A - Perfil espectral da influência do gradiente hidroalcoólico sobre o coeficiente de partição de EPG; B - Espectros de absorção de EPG pelo método de ultrassom submetido a diferentes temperaturas de extração; C -Comparação da porcentagem relativa das três diferentes temperaturas utilizando a média de 5 comprimentos de onda; D Influência do pH no perfil espectral do estrato aquoso de PG para valores de $\mathrm{pH}>8$, evidenciando o dois pontos isobésticos (pi1 e pb2), Ponto isosbéstico: equilíbrio de uma parte protonada e deprotonada da molécula; E Concentração de polifenóis totais em EPG; 
Estes resultados são semelhantes ao encontrado por Carvalho et al. (2008) onde analisou-se o deslocamento espectral em função da hidrólise forçada e formação de pontos isosbésticos em função do $\mathrm{pH}$ em extratos vetais de Brassica sp..

De acordo com Connors (1987) o ponto de intersecção de um grupo de espectros sobrepostos, expressos em função da concentração, evidencia a presença de uma mistura de dois estados e é denominado ponto isosbéstico. Este ponto comprova a presença de uma mistura de dois estados, um protonado e outro deprotonado (Skooget al., 2002). O conhecimento desta informação permite favorecer extrações de classes de constituintes de uma espécie, além de propor uma forma de auxiliar na validação, auxiliando assim na padronização dos extratos em estudos de formulações farmacêuticas posteriores.

Em termos dos resultados da prospecção fitoquími$\mathrm{ca}$, estes revelaram a presença positiva de compostos fenólicos, flavonóides, taninos e cumarinas para todos os extratos hidroalcóolicos analisados (Tabela 4). Contudo, os extratos com alcolaturas acima de 50\% não apresentaram resultados positivos para cumarinas, o que indica uma possível ausência da relação entre as atividades biológicas verificadas com este metabólito. A ausência deste metabólito nestes extratos se justifica pela diferença de polaridade entre o agente extrator e o metabólito em questão.

As plantas são capazes de produzir diferentes substâncias tóxicas em grandes quantidades, como alguns alcaloides, cumarinas e outras provenientes do metabolismo secundário, aparentemente justificado para sua própria defesa contra vírus, bactérias, fungos e animais predadores. Martinez-Florezet al. (2002) relatam que os flavonóides podem atuar na estabilização de espécies reativas de oxigênio, sendo considerados como antioxidantes, possuindo efeito antibacteriano frente a uma grade variedade de cepas. Segundo Sanches et al. (2005), várias espécies de Stryphnodendron sp., como por

Tabela 4 - Resultados da prospecção fotoquímica de Extratos hidroalcóolicos de Piptadeniagonoacantha.

\begin{tabular}{|c|c|c|c|c|c|c|c|c|c|c|c|c|}
\hline \multirow{2}{*}{\multicolumn{2}{|c|}{ Fitoquímica }} & \multicolumn{11}{|c|}{ EPG - gradiente hidroalcoólico (\%) } \\
\hline & & \multirow{2}{*}{$\begin{array}{l}0 \\
-\end{array}$} & \multirow{2}{*}{$\begin{array}{l}10 \\
-\end{array}$} & \multirow{2}{*}{20} & \multirow{2}{*}{30} & \multirow{2}{*}{$\begin{array}{l}40 \\
-\end{array}$} & \multirow{2}{*}{$\begin{array}{l}50 \\
-\end{array}$} & \multirow{2}{*}{$\begin{array}{l}60 \\
-\end{array}$} & \multirow{2}{*}{$\begin{array}{l}70 \\
-\end{array}$} & \multirow{2}{*}{$\begin{array}{l}80 \\
-\end{array}$} & \multirow{2}{*}{$\begin{array}{l}90 \\
-\end{array}$} & \multirow{2}{*}{$\begin{array}{l}100 \\
-\end{array}$} \\
\hline \multirow{3}{*}{ Alcalóides } & Dragendorff & & & & & & & & & & & \\
\hline & Hager & - & - & - & - & - & - & - & - & - & - & - \\
\hline & Mayer & - & - & - & - & - & - & - & - & - & - & - \\
\hline \multirow[b]{2}{*}{ Antraquinona } & Borntrager & - & - & - & - & - & - & - & - & - & - & - \\
\hline & $\begin{array}{l}\text { Acetato de } \\
\text { magnésio }\end{array}$ & + & + & + & + & + & + & + & + & + & + & + \\
\hline \multirow{3}{*}{$\begin{array}{l}\text { Heterosídeos } \\
\text { Cardiotônicos }\end{array}$} & Baljet & - & - & - & - & - & - & - & - & - & - & - \\
\hline & Kedde & - & - & - & - & - & - & - & - & - & - & - \\
\hline & Gelatina salgada & + & + & + & + & + & + & + & + & + & + & + \\
\hline Taninos & $\begin{array}{l}\text { Acetato de } \\
\text { chumbo }\end{array}$ & + & + & + & + & + & + & + & + & + & + & + \\
\hline Cumarinas & $\begin{array}{l}\text { Hidróxido de } \\
\text { potássio }\end{array}$ & + & + & + & + & + & - & - & - & - & - & - \\
\hline $\begin{array}{l}\text { Compostos } \\
\text { fenólicos }\end{array}$ & Fólin-ciocalteau & + & + & + & + & + & + & + & + & + & + & + \\
\hline \multirow{3}{*}{ Flavonóides } & Shinoda & + & + & + & + & + & + & + & + & + & + & + \\
\hline & Cloreto férrico & + & + & + & + & + & + & + & + & + & + & + \\
\hline & $\begin{array}{l}\text { Cloreto de } \\
\text { alumínio }\end{array}$ & + & + & + & + & + & + & + & + & + & + & + \\
\hline \multirow{2}{*}{ Saponinas } & $\begin{array}{l}\text { Índice de } \\
\text { espuma }\end{array}$ & - & - & - & - & + & + & - & - & - & - & - \\
\hline & $\begin{array}{l}\text { Libermann- } \\
\text { burchard }\end{array}$ & - & - & - & - & + & + & - & - & - & - & - \\
\hline
\end{tabular}

(-) ausência; (+) presença. 
exemplo o barbatimão, contém cerca de $20 \%$ de taninos e são usadas na medicina popular como cicatrizantes. Além de agirem como antimicrobianas e desativadoras de radicais livres, ou seja, antioxidantes. Tais espécies são derivados fenólicos, com uma boa solubilidade em água, de massa molecular entre 0,5 a 3 KDA. Muitas vezes, são os princípios ativos de plantas empregadas na medicina tradicional para o tratamento de diversas moléstias, por apresentarem atividades biológicas como a ação bactericida, fungicida, moluscicida e inibição enzimática, que podem ser potencialmente empregadas como novos princípios ativos e/ou outras aplicações (Carvalho et al., 2009; Silva et al., 2004).

Através do método de Folin-Ciocalteau foram quantificados os compostos fenólicos totais (FT) presentes nas amostras de EPG. Os resultados experimentais estão representados na Figura 1 e revelam a concentração de fenóis nos extratos de EPG obtidos através da curva padrão de ácido gálico expressa em equivalente de ácido gálico (EAG/g) no extrato. Esta análise mostrou que este extrato possui grande concentração de compostos fenólicos. Todos os extratos avaliados apresentaram altos teores de compostos fenólicos, quando comparados a dados de outras espécies descritos na literatura (Veliogluet al. 1998; Kahkonen et. al. 1999). Os menores teores de FT foram registrados em EPG a 90 e $100 \%$ $(\mathrm{v} / \mathrm{v})$, sendo os maiores teores encontrados em EPG 30, $40,50,70$ e $80 \%(\mathrm{v} / \mathrm{v})$.

Carvalho et. al. (2010) identificaram, em extratos das folhas desta espécie, dois flavonoides: vitexina e isovitexina. Possivelmente, a presença destes dois flavonoides contribuiria para a elevada concentração de compostos fenólicos no extrato analisado.

Com relação à atividade antibacteriana de extratos vegetais não existe um consenso sobre o nível aceitável quando comparados com antibióticos padrões. Alguns

Tabela 5. Eficiência relativa de extratos de P. gonoacanthafrente à Staphylococcus aureus em relação AERITROMICINA.

\begin{tabular}{c|cc|cc}
\hline \multirow{2}{*}{$\begin{array}{c}\text { Graduação } \\
\text { alcoólica do } \\
\text { extrato (\% v/v) }\end{array}$} & \multicolumn{4}{|c}{ Concentração do extrato } \\
\cline { 2 - 5 } & ${ }^{*}$ Halos $(\mathrm{mm})$ & $\begin{array}{c}* * \text { Eficiência } \\
(\%)\end{array}$ & Halos ${\mathrm{mg} \cdot \mathrm{mL}^{-1}}^{(\mathrm{mm})}$ & $\begin{array}{c}\text { Eficiência } \\
(\%)\end{array}$ \\
\cline { 2 - 5 } & $7,10 \pm 0,2828$ & 44,40 & $10,20 \pm 0,1414$ & 63,80 \\
\hline $\mathbf{0}$ & $7,25 \pm 0,1768$ & 45,30 & $10,25 \pm 0,5303$ & 64,10 \\
\hline $\mathbf{1 0}$ & $7,50 \pm 0,4243$ & 46,90 & $11,00 \pm 1,061$ & 68,80 \\
\hline $\mathbf{2 0}$ & $8,50 \pm 0,3536$ & 53,10 & $11,00 \pm 0,1414$ & 68,80 \\
\hline $\mathbf{3 0}$ & $8,50 \pm 0,3536$ & 53,10 & $11,50 \pm 1,061$ & 71,90 \\
\hline $\mathbf{5 0}$ & $9,00 \pm 0,3536$ & 56,30 & $11,50 \pm 0,3536$ & 71,90 \\
\hline $\mathbf{6 0}$ & $10,00 \pm 0,7071$ & 62,50 & $12,75 \pm 0,5303$ & 79,70 \\
\hline $\mathbf{7 0}$ & $10,25 \pm 0,1768$ & 64,10 & $13,00 \pm 0,3536$ & 81,30 \\
\hline $\mathbf{9 0}$ & $10,75 \pm 1,237$ & 67,20 & $13,50 \pm 0,3536$ & 84,40 \\
\hline $\mathbf{1 0 0}$ & $8,50 \pm 0,3536$ & 53,10 & $11,00 \pm 0,7071$ & 68,80 \\
\hline$* * *+$ & $7,50 \pm 0,3536$ & 46,90 & $10,00 \pm 0,5657$ & 62,50 \\
\hline
\end{tabular}

*Diâmetros médios em mm; **Eficiência relativa ao controle positivo; ${ }^{* * *} \mathrm{C}+$, Controle positivo com Eritromicina a $10 \mu \mathrm{g} \cdot \mu \mathrm{L}-1$. 

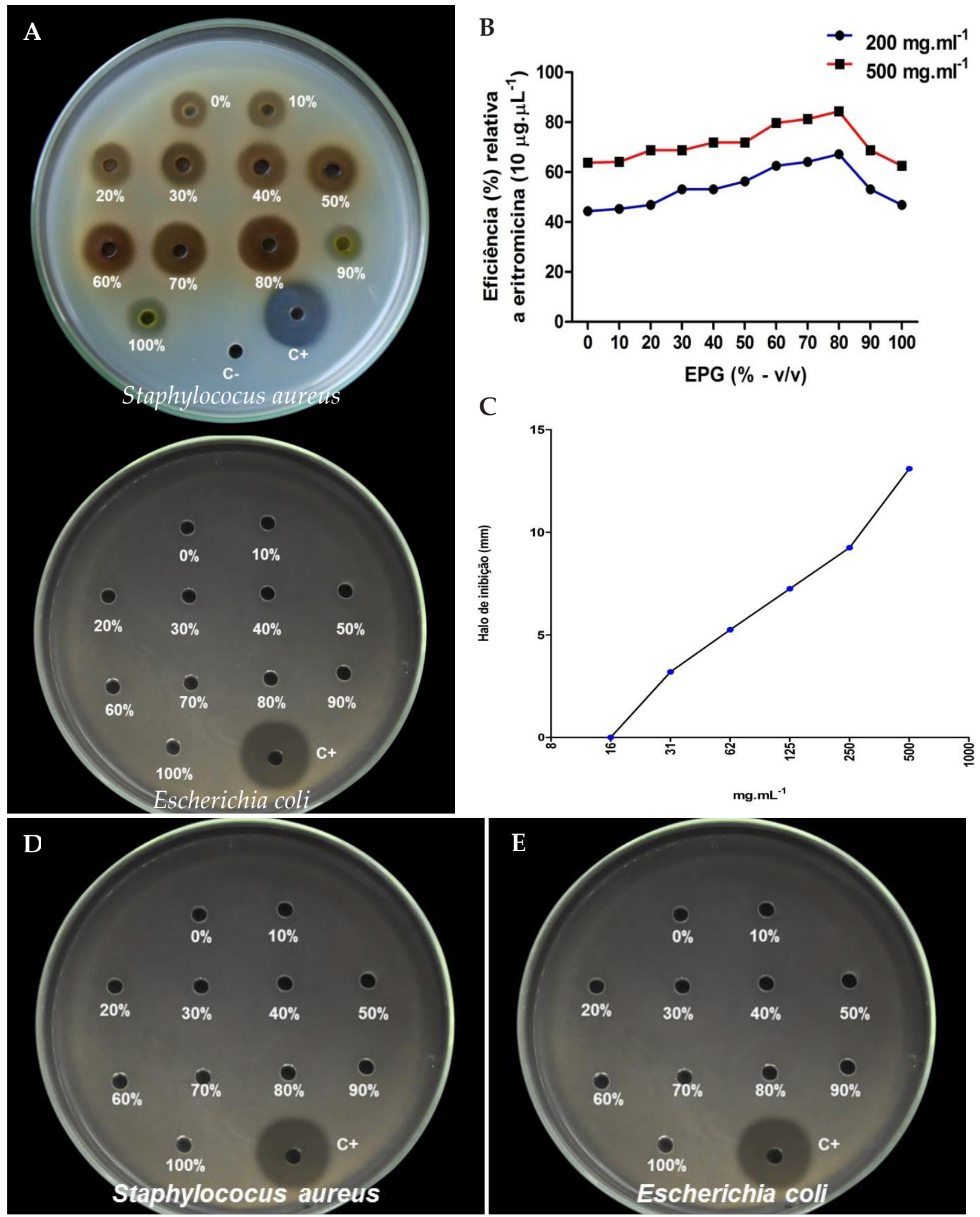

Figura 2. Avaliação da atividade antibacteriana de extratos de folhas de Piptadeniagonoacantha(EPG). A Atividade antibacteriana de EPG em diferentes graduações alcoólicas na concentração de 500 mg.mL-1 frente a S. aureus e E. Coli; B -Comparação do tamanho do halo de inibição apresentado pelas diferentes concentrações (200 e 500 mg.mL-1) utilizando EPG em diferentes graduações alcoólicas frente a S. aureus; C - Concentração inibitória mínima de EPG a 80 \% (v/v); E e F - Ausência de halo de inibição quando utilizado apenas os solventes em diferentes graduações alcóolicas. C+, Eri - Eritromicina a $10 \mu \mathrm{g} . \mu \mathrm{L}-1$ e Cip - Ciprofloxacino0, $1 \mu \mathrm{g} . \mu \mathrm{L}-1$. 
autores consideram somente resultados similares aos de antibióticos conhecidos, desde que se trabalhe com uma fração já determinada (Aligianniset al., 2001). Entretanto, como este trabalho foi realizado com frações hidroalcóolicas de P.gonoacantha, seguiu-se o critério sugerido por Holetzet al. (2002), considerando como resultado final de cada extrato a média das três medidas dos halos de inibição e como sensível halo igual ou acima de oito mm de diâmetro conforme critérios deParekh e Chanda (2007); e Santos et. al. (2007). O volume mínimo de extrato hidroalcoólico utilizado baseou-se em estudos anteriores, realizados em nosso laboratório, que apontaram o valor de $10 \mu \mathrm{L}$ como a menor quantidade de extrato necessária para verificar a atividade antibacteriana em placa pelo método de difusão (Miranda et. al., 2013).

Todos os extratos das folhas de P. gonoacantha avaliados apresentaram atividade frente à $S$. aureus. Porém, na concentração de 200 mg.mL-1 os EPG a 10 e $20 \%$, não atingiram o tamanho do halo necessário para classificá-los como efetivos. Os resultados das respectivas médias dos diâmetros dos halos de inibição e sua eficácia, quando comparada ao controle positivo, podem ser visualizados na Tabela 5 .

O extrato que apresentou melhor atividade foi o com graduação alcoólica de $80 \%$ (v/v) (Figura 2A). Nota-se que a atividade antibacteriana do extrato utilizando os diversos solventes mantém um perfil, sendo os resultados na concentração de 500 mg.mL-1 mais expressivos em relação ao mesmo extrato a 200 mg.mL-1 (Figura 2B). Além deste, foi realizada a análise da CIM, onde se considerou como CIM a menor concentração dos extratos que inibiu completamente o crescimento de $S$. aureus. O extrato hidroalcoólico (80\%) de P. gonoacantha apresentou uma CIM igual a 31,25 mg.mL-1 (Figura 2C). Quanto à atividade frente à bactéria Escherichia coli nenhum dos extratos estudados apresentaram atividade inibitória (Figura 2B).

Com relação ao teste realizado a fim de verificar a atividade bactericida do álcool utilizado para a preparação dos extratos, nas diferentes graduações alcoólicas (0 a 100\% - v/v), todos os resultados foram negativos, exceto o controle positivo (Figura $2 \mathrm{~d}$ e E). A não inibição do crescimento com o controle mostra que o etanol não exerceu influência sobre os resultados da atividade do extrato, resultado já descrito por Virtuoso et al. (2005). Assim ficou evidenciado que a função do álcool foi apenas de possibilitar a extração e veiculação do(s) composto(s) dotado(s) de atividade antibacteriana. Tal resultado atribui-se ao fato do álcool volatilizar rapidamente, não interferindo no crescimento dos microrganismos.

O composto galato de metila tem sido isolado de várias plantas como derivado do ácido gálico e apresenta atividade antibacteriana e antiviral (Meyre-Silva et. al., 2001). Este composto foi isolado de folhas desta espécie e pode estar relacionado à atividade antibacteriana exposta. Além deste, alguns flavonoides, como vitexina e isovitexina, também foram isolados desta espécie
(Carvalho et al., 2010).

Os flavonóides são compostos fenólicos de vasta ocorrência na natureza, desempenhando diversas funções fisiológicas nos vegetais. Na família Fabaceae estes metabólitos secundários cumprem, entre outras funções, o papel de antimicrobianos. Souza Filho (2004) demonstrou a atividade antibacteriana do flavonoide ramnosil-O-vitexina isolado da espécie LupinuslanatuscontraS. aureus, Klebsiellapneumoniae e Escherichia coli. Portanto, sendo as espécies Lupinuslanatus e Piptadeniagonoacanthapertencentes à mesmafamília, Fabaceae, pode-se inferir que tais compostos podem estar envolvidos na atividade antibacteriana apresentada.

A ação antimicrobiana dos flavonoides, provavelmente, está relacionada à capacidade de complexar proteínas extracelulares e solúveis, bem como estruturas da parede celular. Além disso, alguns flavonoides mais lipofílicos podem atuar provocando o rompimento de membranas microbianas (Cowan, 1999).

\section{Considerações finais}

Esses resultados colaboram na elucidação preliminar de que os compostos presentes nos extratos das folhas de Piptadeniagonoacantha, especialmente os já identificados (galato de metila, vitexina e isovitexina), estão envolvidos na atividade biológica testada, podendo futuramente direcionar novos estudos.

Cabe ressaltar que o uso abusivo e indiscriminado de agentes antimicrobianos na prática clínica humana e veterinária tem um efeito seletivo no surgimento e manutenção de resistência a drogas. Uma tentativa de manter o uso de antimicrobianos atuais poderia ser encontrada em sua combinação com outros produtos, como os produtos naturais, que representariam uma opção terapêutica no tratamento de infecções causadas por S. aureus e outros patógenos, no que diz respeito ao aparecimento crescente de resistência múltipla.

\section{Referências}

Aligiannis N, Kalpoutzakis E, Mitaku S, ChinouIB. Composition and antimicrobial activity of the essential oils of two Origanumspecies. JAgricFoodChem 2001; 49 (9):4168-70.

Amato Neto V, Nicodemo AC, Lopes HV. Antibióticos na Prática Médica. São Paulo: Roca; 1994. 283 p.

Brasil. Resolução de Diretoria Colegiada (RDC) In: ANVISA., editor. Ministério da Saúde. . v. 313 Series Resolução de Diretoria Colegiada (RDC) 2005.

Carvalho CA, Fernandes KM, Matta SL, Silva MB 
da, Oliveira LL de, Fonseca CC. Evaluation of antiulcerogenic activity of aqueous extract of Brassica oleraceavar.capitata (cabbage) on Wistar rat gastric ulceration. ArqGastroenterol 2011; 48 (4):276-82.

Carvalho CA, Matta SLP, Melo FCSA, Andrade DCF, Carvalho LM, Nascimento PC, Silva MB da, Rosa MB da Rosa. Stem herb (Tynnanthusfasciculatus MIERS- Bignoniaceae): phytochemical and toxicological study involving Artemia. Revista Eletrônica de Farmácia 2009; 6 (1):51 - 8.

Carvalho CA, Silva MB, Oliveira

TG, Lima JM, Rosa MB

ometricstudyatdifferentphenologicstagesofthecabbage (Brassicaoleraceae var. capitata).

BrazilianJournalofPharmacognosy 2008; 18 (2):249 - 57.

Carvalho MG, Cardozo MA, Catunda Junior FE, Catunda Junior FEA, Carvalho AG. ChemicalconstituentsofPiptadeniagonoacantha J.F. Macbr. Anais da Academia Brasileira de Ciências 2010; 82 (3):561-7.

Connors KA. Binding Constants: The Measurement of Molecular Complex Stability. New York: WileyInterscience; 1987. $432 \mathrm{p}$.

Cowan MM. Plant products as antimicrobial agents. ClinMicrobiolRev 1999; 12 (4):564-82.

Falkenberg MB, Santos RI, Simões CMO. Introdução à análise fitoquímica. In: $5^{\underline{a}}$, editor, translatorand editor Farmacognosia: da planta ao medicamento. Florianópolis: Editora da UFRGS; 2003; p. 230-4.

Ferri PH. Química de produtos naturais: métodos gerais. In: LC DI STASI, editor, translatorand editor Plantas Medicinais: arte e ciência: Um guia de estudo interdisciplinar. São Paulo: Unesp; 1996; p. $130-6$.

Francony A, Pétrier C. Sonochemical degradation of carbon tetrachloride in aqueous solution at two frequencies: $20 \mathrm{kHz}$ and $500 \mathrm{kHz}$. UltrasonicsSonochemistry 1996; 3 (2):77 - 82.

Holetz FB, Pessini GL, Sanches NR, Cortez DAG Cortez, Nakamura CV, Dias Filho BP. Screening of some plants used in the Brazilian folk medicine for the treatment of infectious diseases. MemInstOswaldo Cruz 2002; 97 (7):1027-31.

Kähkönen MP, Hopia AI, Vuorela HJ, Rauha JP, Pihlaja K, Kujala TS, Heinonen M. Antioxidant activity of plant extracts containing phenolic compounds. J Agric Food Chem 1999; 47 (10):395462.

Korn M, Pereira MG, Borges SS. Algumas Aplicações Analíticas dos Ultra-sons. Boletim da Sociedade Portuguesa de Química 2005; 96:51 - 6.

Martínez-Flórez S, González-Gallego J, Culebras JM, Tuñón MJ. Flavonoids: properties and antioxidizing action. NutrHosp 2002; 17 (6):271-8.

Meyre-Silva C, Yunes RA, DelleMonache F, Santos AR, Schmeling LO, Gadotti VM, Liz F, CechinelFilho V. Phytochemical and pharmacological analysis of Bauhinia microstachya (Raddi) Macbr. (Leguminosae). Z Naturforsch C 2001; 56 (1112):939-42.

Miranda GS, Santana GS, Machado BB, Coelho FP, Carvalho CA. Atividade antibacteriana in vitro de quatro espécies vegetais em diferentes graduações alcoólicas. Revista Brasileira de Plantas Medicinais 2013; 15 (1): Prelo.

Nascentes CC, Korn M, Arruda MAZ. A fast ultrasound-assisted extraction of $\mathrm{Ca}, \mathrm{Mg}, \mathrm{Mn}$ and Zn from vegetables.Microchemical Journal 2001; 69 (1):37 - 43 .

NCCLS.Performance standards for antimicrobial disk susceptibility tests. Approved standard, 8th ed. NCCLS document M2-A8. NCCLS, Wayne, Pa. In., Series Performance standards for antimicrobial disk susceptibility tests. Approved standard, 8th ed. NCCLS document M2-A8. NCCLS, Wayne, Pa.; 2003.

NCCLS.Performance standards for antimicrobial susceptibility testing. Twelfth informational supplement. NCCLS document M100-S12. NCCLS, Wayne, Pa. 2002.

Parekh J, Chanda SV. In vitro antimicrobial activity and phytochemical analysis of some Indian medicinal plants.TurkishJournalofBiology 2007; 31:53 - 8 .

Peres RL, Moraes SCS, Carvalho CA, Nascimento PC, Carvalho LM, Silva MB da, Rampelotto $\mathrm{PH}$, Rosa MB da.Achilleamillefolium Asteraceae: Phytochemical, antifungal activity (Colletotrichummusae) and spectrophotometric study. Revista Eletrônica de Farmácia 2009; 6 (3):81 $-93$.

Pinelo M, Rubilar M, Jerez M, Sineiro J, Núñez MJ. 
Effect of solvent, temperature, and solvent-to-solid ratio on the total phenolic content and antiradical activity of extracts from different components of grape pomace.JAgricFoodChem 2005; 53 (6):2111-7.

Ribeiro MC, Soares MMSR. Microbiologia prática: roteiro e manual. 2 ed. São Paulo: Atheneu; 2008.

Ruiz-Jiménez J, Luque-García JL, Castro MDL. Dynamic ultrasound-assisted extraction of cadmium and lead from plants prior to electrothermal atomic absorption spectrometry. AnalyticaChimica Acta2003; 480:231 - 7.

Sanches ACC, Lopes GC, Nakamura CV, Dias Filho BP, Mello JCP. Antioxidant and antifungal activities of extracts and condensed tannins from StryphnodendronobovatumBenthRevistaBrasileira de CiênciasFarmacêuticas2005; 41 (1):101 - 7.

Santos SC, Ferreira FS, Rossi-Alva JC, Fernandez LG. "In vitro antimicrobial activity of the extract of Abaremacochliocarpos (Gomes) Barneby\& Grimes. BrazilianJournalofPharmacognosy 2007; 17 (2):215 -9 .

Silva HH, Silva IG, dos Santos RM, Rodrigues Filho E, Elias CN. Larvicidal activity of tannins isolated of Magoniapubescens St. Hil. (Sapindaceae) against Aedesaegypti (Diptera, Culicidae. Brazilian Society for Tropical Medicine 2004; 37 (5):396 - 9.

Simões CMO, Schnkel EP, Gosmann G, Mello JCP, Mentz LA, Petrovick PR, 2004. Farmacognosia: da planta ao medicamento. Editora da UFRGS Florianópolis.

Skoog DA, Holler FJ, Nieman TA. Principles of instrumental analysis. $5^{\mathrm{a}}$, editor. PhiladelphiaSaundersCollegePublishing; 2002.(5 editor).

Souza Filho PAC. Estudo químico e biológico em LupinuslanatusBentham (Leguminosae-Faboideae) [Dissertação (mestrado)]. Porto Alegre: UFRGS; 2004. 100 p.

Spigno G, Tramelli L, Faveri DM. Effects of extraction time, temperature and solvent on concentration and antioxidant activity of grape marc phenolics. Journal of Food Engineering 2007; 81 (1):200 - 8.

Suslick KS. Sonochemistry.Science 1990; 247 (4949):1439-45.

Vale MGR, luz LP, Martins AF, Caramão EB, Dariva C, Oliveira JV. Extraction of organic material in mineral coal by using supercritical fluid extraction, soxhlet, and sonication methods. Journal of Microcolumn Separations 1998; 10 (3):259 - 63.

Velioglu YS, Mazza G, Gao L, Oomah BD. Antioxidant Activity and Total Phenolics in Selected Fruits, Vegetables, and Grain Products Journal of Agricultural and Food Chemistry 1998; 46 (10): $4113-7$.

Vinatoru M, Toma M, Radu O et al. The use of ultrasound for the extraction of bioactive principles from plant materials. UltrasonSonochem 1997; 4 (2):135-9.

Virtuoso S, Davet A, Dias JF, Cunico MM, Miguel MD, Oliveira AB, Miguel OG. Preliminary study of the antibacterial activity of ErythrinavelutinaWilld. Fabaceae(Leguminosae) bark.Brazilian Journal of Pharmacognosy 2005; 15 (2):137 - 42.

Yilmaz Y, Toledo RT. Oxygen radical absorbance capacities of grape/wine industry byproducts and effect of solvent type on extraction of grape seed polyphenols. Journal of Food Composition and Analysis 2006; 19 (1):41 - 4.

ctrometricstudyatdifferentphenologicstagesofthecabbage (Brassicaoleraceae var. capitata).

BrazilianJournalofPharmacognosy 2008; 18 (2):249 $-57$.

Carvalho MG, Cardozo MA, Catunda Junior FE, Catunda Junior FEA, Carvalho AG. ChemicalconstituentsofPiptadeniagonoacantha J.F. Macbr. Anais da Academia Brasileira de Ciências 2010; 82 (3):561-7.

Connors KA. Binding Constants: The Measurement of Molecular Complex Stability. New York: WileyInterscience; 1987. $432 \mathrm{p}$.

Cowan MM. Plant products as antimicrobial agents. ClinMicrobiolRev 1999; 12 (4):564-82.

Falkenberg MB, Santos RI, Simões CMO. Introdução à análise fitoquímica. In: 5aㅡ, editor, translatorand editor Farmacognosia: da planta ao medicamento. Florianópolis: Editora da UFRGS; 2003; p. 230-4.

Ferri PH. Química de produtos naturais: métodos gerais. In: LC DI STASI, editor, translatorand editor Plantas Medicinais: arte e ciência: Um guia de estudo interdisciplinar. São Paulo: Unesp; 1996; p. $130-6$.

Francony A, Pétrier C. Sonochemical degradation 
of carbon tetrachloride in aqueous solution at two frequencies: $20 \mathrm{kHz}$ and $500 \mathrm{kHz}$. UltrasonicsSonochemistry 1996; 3 (2):77 - 82.

Holetz FB, Pessini GL, Sanches NR, Cortez DAG Cortez, Nakamura CV, Dias Filho BP. Screening of some plants used in the Brazilian folk medicine for the treatment of infectious diseases. MemInstOswaldo Cruz 2002; 97 (7):1027-31.

Kähkönen MP, Hopia AI, Vuorela HJ, Rauha JP, Pihlaja K, Kujala TS, Heinonen M. Antioxidant activity of plant extracts containing phenolic compounds. J Agric Food Chem 1999; 47 (10):395462.

Korn M, Pereira MG, Borges SS. Algumas Aplicações Analíticas dos Ultra-sons. Boletim da Sociedade Portuguesa de Química 2005; 96:51 - 6.

Martínez-Flórez S, González-Gallego J, Culebras JM, Tuñón MJ. Flavonoids: properties and antioxidizing action. NutrHosp 2002; 17 (6):271-8.

Meyre-Silva C, Yunes RA, DelleMonache F, Santos AR, Schmeling LO, Gadotti VM, Liz F, CechinelFilho V. Phytochemical and pharmacological analysis of Bauhinia microstachya (Raddi) Macbr. (Leguminosae). Z Naturforsch C 2001; 56 (1112):939-42.

Miranda GS, Santana GS, Machado BB, Coelho FP, Carvalho CA. Atividade antibacteriana in vitro de quatro espécies vegetais em diferentes graduações alcoólicas. Revista Brasileira de Plantas Medicinais 2013; 15 (1): Prelo.

Nascentes CC, Korn M, Arruda MAZ. A fast ultrasound-assisted extraction of $\mathrm{Ca}, \mathrm{Mg}, \mathrm{Mn}$ and Zn from vegetables.Microchemical Journal 2001; 69 (1):37 - 43 .

NCCLS.Performance standards for antimicrobial disk susceptibility tests. Approved standard, 8th ed. NCCLS document M2-A8. NCCLS, Wayne, Pa. In., Series Performance standards for antimicrobial disk susceptibility tests. Approved standard, 8th ed. NCCLS document M2-A8. NCCLS, Wayne, Pa.; 2003.

NCCLS.Performance standards for antimicrobial susceptibility testing. Twelfth informational supplement. NCCLS document M100-S12. NCCLS, Wayne, Pa. 2002.

Parekh J, Chanda SV. In vitro antimicrobial activity and phytochemical analysis of some Indian medicinal plants.TurkishJournalofBiology 2007; $31: 53-8$.

Peres RL, Moraes SCS, Carvalho CA, Nascimento PC, Carvalho LM, Silva MB da, Rampelotto $\mathrm{PH}$, Rosa MB da.Achilleamillefolium Asteraceae: Phytochemical, antifungal activity (Colletotrichummusae) and spectrophotometric study. Revista Eletrônica de Farmácia 2009; 6 (3):81 $-93$.

Pinelo M, Rubilar M, Jerez M, Sineiro J, Núñez MJ. Effect of solvent, temperature, and solvent-to-solid ratio on the total phenolic content and antiradical activity of extracts from different components of grape pomace.JAgricFoodChem 2005; 53 (6):2111-7.

Ribeiro MC, Soares MMSR. Microbiologia prática: roteiro e manual. 2 ed. São Paulo: Atheneu; 2008.

Ruiz-Jiménez J, Luque-García JL, Castro MDL. Dynamic ultrasound-assisted extraction of cadmium and lead from plants prior to electrothermal atomic absorption spectrometry. AnalyticaChimica Acta2003; 480:231 - 7.

Sanches ACC, Lopes GC, Nakamura CV, Dias Filho BP, Mello JCP. Antioxidant and antifungal activities of extracts and condensed tannins from StryphnodendronobovatumBenthRevistaBrasileira de CiênciasFarmacêuticas2005; 41 (1):101 - 7.

Santos SC, Ferreira FS, Rossi-Alva JC, Fernandez LG. "In vitro antimicrobial activity of the extract of Abaremacochliocarpos (Gomes) Barneby\& Grimes. BrazilianJournalofPharmacognosy 2007; 17 (2):215 $-9$.

Silva HH, Silva IG, dos Santos RM, Rodrigues Filho E, Elias CN. Larvicidal activity of tannins isolated of Magoniapubescens St. Hil. (Sapindaceae) against Aedesaegypti (Diptera, Culicidae. Brazilian Society for Tropical Medicine 2004; 37 (5):396 - 9.

Simões CMO, Schnkel EP, Gosmann G, Mello JCP, Mentz LA, Petrovick PR, 2004. Farmacognosia: da planta ao medicamento. Editora da UFRGS Florianópolis.

Skoog DA, Holler FJ, Nieman TA. Principles of instrumental analysis. $5^{\text {a }}$, editor.

PhiladelphiaSaundersCollegePublishing; 2002.(5 $5^{\text {a }}$ editor).

Souza Filho PAC. Estudo químico e biológico em LupinuslanatusBentham (Leguminosae-Faboideae) [Dissertação (mestrado)]. Porto Alegre: UFRGS; 
2004. 100 p.

Spigno G, Tramelli L, Faveri DM. Effects of extraction time, temperature and solvent on concentration and antioxidant activity of grape marc phenolics. Journal of Food Engineering 2007; 81 (1):200 - 8.

Suslick KS. Sonochemistry.Science 1990; 247 (4949):1439-45.

Vale MGR, luz LP, Martins AF, Caramão EB, Dariva C, Oliveira JV. Extraction of organic material in mineral coal by using supercritical fluid extraction, soxhlet, and sonication methods. Journal of Microcolumn Separations 1998; 10 (3):259 - 63.

Velioglu YS, Mazza G, Gao L, Oomah BD. Antioxidant Activity and Total Phenolics in Selected Fruits, Vegetables, and Grain Products Journal of Agricultural and Food Chemistry 1998; 46 (10): $4113-7$.

Vinatoru M, Toma M, Radu O et al. The use of ultrasound for the extraction of bioactive principles from plant materials. UltrasonSonochem 1997; 4 (2):135-9.

Virtuoso S, Davet A, Dias JF, Cunico MM, Miguel MD, Oliveira AB, Miguel OG. Preliminary study of the antibacterial activity of ErythrinavelutinaWilld. Fabaceae(Leguminosae) bark.Brazilian Journal of Pharmacognosy 2005; 15 (2):137 - 42.

Yilmaz Y, Toledo RT. Oxygen radical absorbance capacities of grape/wine industry byproducts and effect of solvent type on extraction of grape seed polyphenols. Journal of Food Composition and Analysis 2006; 19 (1):41 - 4. 